\title{
2.6 PULSAR NP 0532: RECENT RESULTS ON STRONG PULSES OBTAINED AT ARECIBO
}

\author{
CARL HEILES* \\ Arecibo Observatory, Arecibo, Puerto Rico \\ and \\ JOHN M. RANKIN \\ Department of Physics and Astronomy, University of Iowa, Iowa City, Iowa, U.S.A.
}

\begin{abstract}
New observations have been made of strong pulses from NP 0532. Linear polarization is typically 25 per cent and circular polarization is less than 10 per cent but definitely not zero. A comparison of pulse shapes at 429.9 and $430.1 \mathrm{MHz}$ showed significant differences in detailed structure and polarization. In another study strong pulses at 318 and $111 \mathrm{MHz}$ did not occur together although a strong pulse at one frequency was accompanied by an above average intensity at the other frequency. Similar behaviour was found at 111 and $74 \mathrm{MHz}$. We conclude that strong pulses do not have a smooth continuous frequency spectrum.
\end{abstract}

\section{Clarification of Previously Published Results}

The properties of strong pulses from NP 0532 have been investigated by Heiles et al. (1970) at $430 \mathrm{MHz}$ using a bandwidth of $8 \mathrm{kHz}$. They found that the pulses were sometimes $₹ 100 \mu \mathrm{sec}$ in width and showed circular polarization whose sense varied systematically with the arrival time of the strong pulse within the average pulse envelope, whose half-width is about $250 \mu \mathrm{sec}$. However, observations with a $300 \mathrm{kHz}$ bandwidth (which yields a time resolution of about $2 \mathrm{msec}$ due to dispersion) by Graham et al. (1970) showed little circular polarization but did show 70 per cent linear polarization in a typical pulse. We have performed two new sets of observations to clarify the situation.

One observation used $300 \mathrm{kHz}$ bandwidth and a time constant of $1 \mathrm{msec}$, and was basically a repetition of the work done at Jodrell Bank (Graham et al., 1970). These observations have been published elsewhere (Rankin and Heiles, 1970) and will not be described in detail here. Only strong main pulses were observed - no precursors or interpulses. Most of the strong pulses did not show the 70 percent linear polarization implied to be typical by Graham et al. (1970). Linear polarizations of order 25 per cent were typical but many pulses showed much smaller polarization. There was no systematic trend in position angle from one pulse to another. Circular polarization was nearly always smaller than linear polarization, usually less than 10 per cent, but definitely not always zero.

The other observation was done on 11 July 1970, and used both senses of circular polarization, recording each simultaneously in two bandwidths centered at precisely 429.9 and $430.1 \mathrm{MHz}$. Each of the four channels was mixed to baseband and the

* Usual address: Department of Astronomy, University of California, Berkeley. 
bandwidths were defined by $R C$ time constants which provided $3 \mathrm{~d} B$ total widths of about $7.5 \mathrm{kHz}$, or integrated bandwidths of $12 \mathrm{kHz}$. The signals were then detected, passed through approximately rectangular $100 \mu \mathrm{sec}$ time filters, and recorded at $50 \mu \mathrm{sec}$ intervals in synchronism with the pulsar period.

As is our usual practice, we computed the energy distribution on and off the main pulse; the strong pulses were selected on the basis of these distributions and were positively substantiated by detection with approximately the correct time delay at both frequencies. Previous $430 \mathrm{MHz}$ distributions have been given previously (Heiles et al., 1970; Rankin and Heiles, 1970), and we will not repeat them here.

The average of all the strong pulses was obtained and is shown in Figure 1. It does

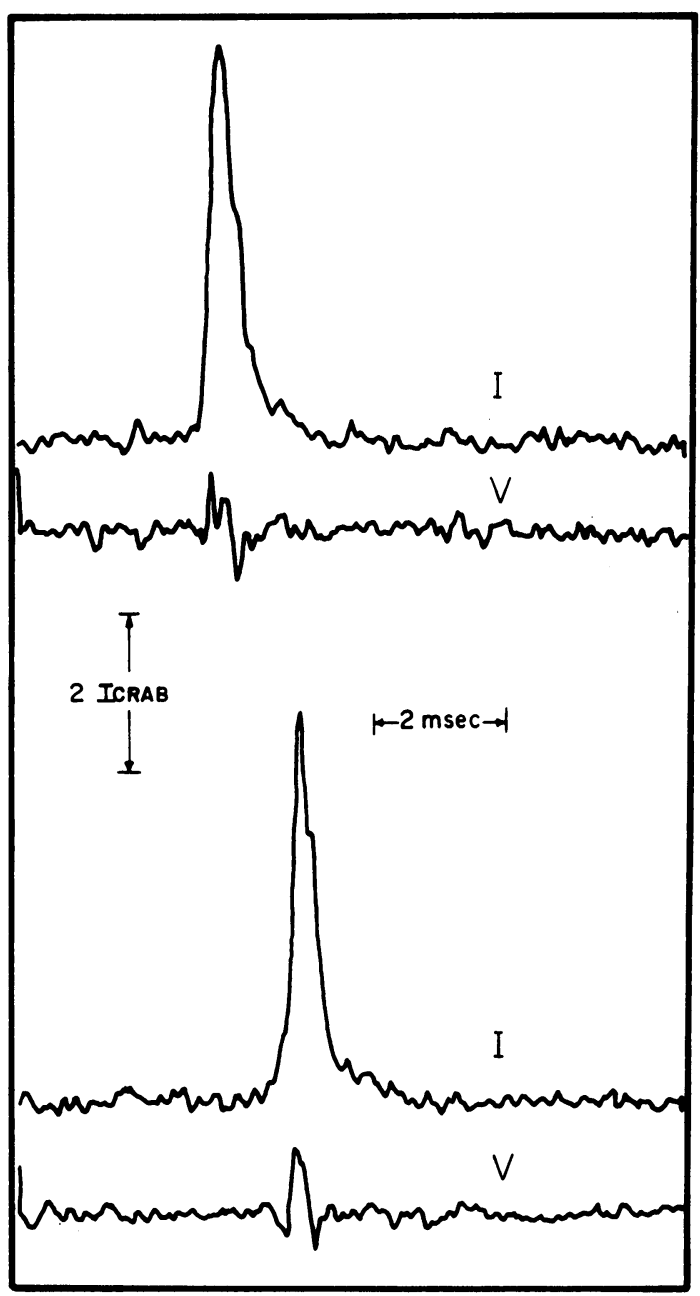

Fig. 1. Average of the strong pulses at $430.1 \mathrm{MHz}$ (top) and $429.9 \mathrm{MHz}$ (bottom) in total intensity, I and circularly-polarized Stokes parameter, $V$. The relative time delay due to dispersion is evident. Note the absence of circular polarization reported earlier! 
not show the circular polarization reversal that was found earlier (Heiles et al., 1970). We know of no reason why there should be any systematic effects in either experiment. We can only conclude that our previous result was a statistical fluke due to the small number of pulses involved.

Individual strong pulses appeared different in the two channels. When the pulse was narrow, it would often arrive at different phases of the pulsar period at the two frequencies. Also, differences in pulse width were common and the sense of circular polarization was often different. Examples can be seen in Figure 2.

Some differences in pulse shapes observed at the two frequencies are clearly expected because the time-bandwidth product for each plotted point in Figure 2 is only about 1.2. However, we question whether this can account for the differences

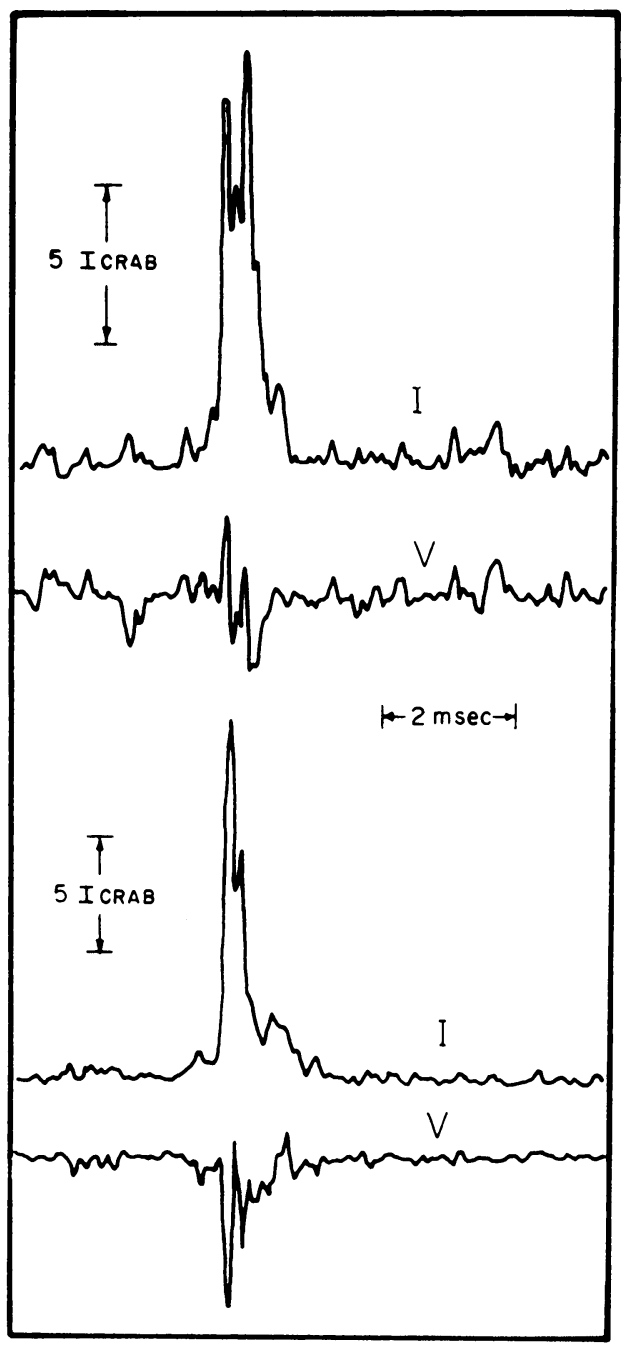

Fig. 2a. 
in pulse width we often observe, or for the differences in circular polarization, especially in cases where the pulse is wide enough to substantially increase the effective timebandwidth product. Furthermore, in the cases where the pulse or its components are narrow, they should at least arrive at the same pulsar phase at the two frequencies.

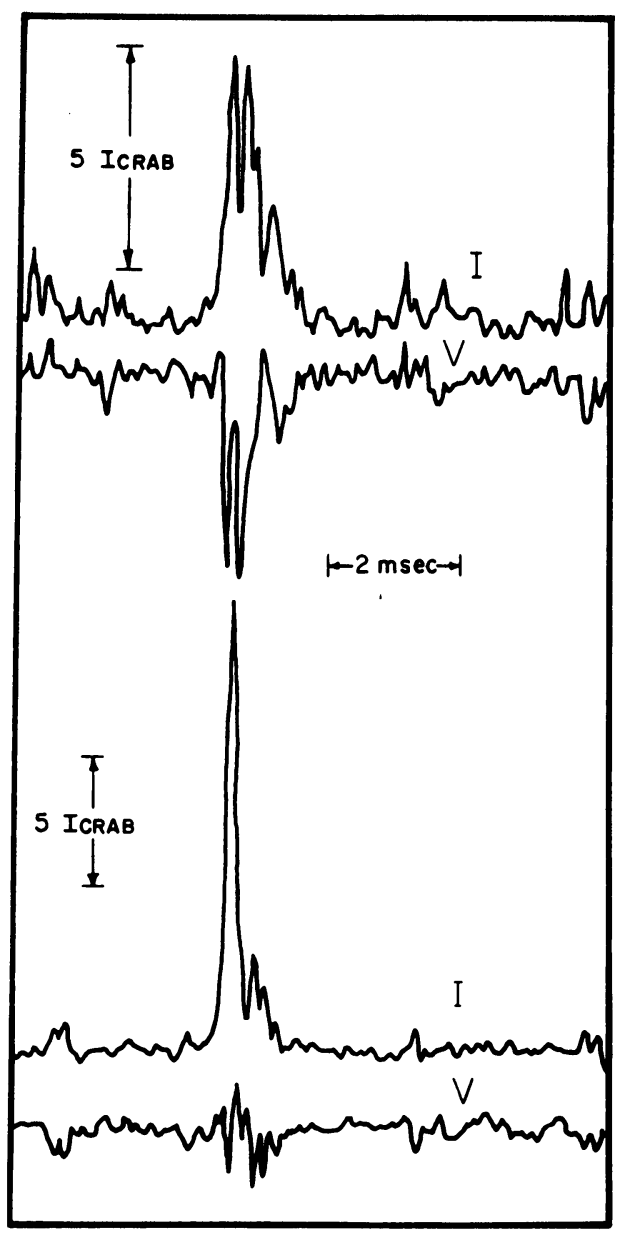

Fig. 2b.

This is often not the case. Therefore the pulse shape differences are thought to be significantly real. However, further measurements are obviously called for and will be performed in the near future.

\section{New Observational Results}

Most of our new observations are being published elsewhere (Heiles and Rankin, 1971; Rankin and Heiles, 1971b) and here we will only summarize the results. The 
new results involve simultaneous observations at various frequencies and an approximate determination of the spectral behavior of strong pulses.

Simultaneous observations of individual strong pulses at 318 and $111 \mathrm{MHz}$ reveal that a detectable strong pulse at one frequency does not usually give rise to an independently detectable strong pulse at the other frequency. However, a strong pulse detected at one frequency does tend to produce a pulse which is stronger than the average pulse intensity at the other frequency. A strong pulse which is detected at

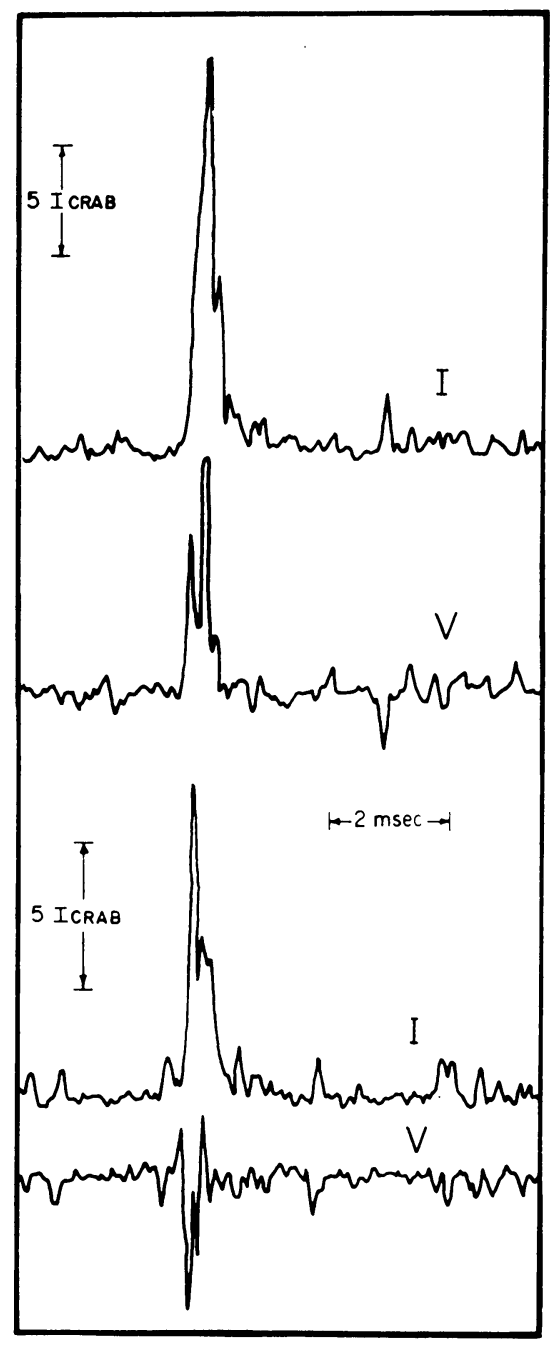

Fig. 2c.

Fig. 2a-c. Individual $430 \mathrm{MHz}$ strong pulses at $430.1 \mathrm{MHz}$ (top) and $429.9 \mathrm{MHz}$ (bottom) in total intensity, $I$, and circularly-polarized Stokes parameter, $V$. The relative time delay due to dispersion has been removed by shifting the graphs by the appropriate amount. The intensity scales for $V$ and $I$ at each frequency are the same, but the scales at the two frequencies are different and are shown.

Note the different pulse shapes and widths, and the differences in $V$ at the two frequencies. 
$318 \mathrm{MHz}$ tends to produce a pulse at $111 \mathrm{MHz}$ which is about eight times stronger than the average $111 \mathrm{MHz}$ pulse, and vice versa. However, detectable strong pulses at any frequency are typically 1500 times stronger than the average pulse. Thus there is a discrepancy of more than two orders of magnitude which can only be the result of real frequency structure in the spectrum of an individual strong pulse.

Similar behavior is exhibited between 111 and $74 \mathrm{MHz}$ as revealed by further simultaneous measurements. About half of the strong pulses are independently detectable at both frequencies, but the other half behave in a manner akin to the usual one seen in the $318-111 \mathrm{MHz}$ data described above. These data lead to the conclusion that the strong pulses are not received with a smooth, continuous frequency spectrum. Our 111-74 MHz observations imply a frequency width of about $30 \mathrm{MHz}$, perhaps implying that

$$
\Delta f \mid f \approx \frac{1}{3} \text {. }
$$

On the other hand, the spectral behavior may be more complicated. Further observations are required to determine the spectral behavior more accurately. Additional spectral information is given in our other papers (Heiles and Rankin, 1971).

These observations were obtained with large bandwidths and do not suffer from a small time-bandwidth product. Current knowledge about interstellar scintillation (Heiles and Rankin, 1971) allows us to rule out interstellar scintillation as a significant contributing cause to the spectral behavior described here. The pulse emission mechanism itself, or scattering near the pulsar itself, could be responsible for the frequency behavior. In any case, the existence of occasional huge pulses which are characterized by complex spectral behavior and random polarization behavior contrasts with other pulsars, which are not thought to show these properties.

\section{Acknowledgement}

The Arecibo Observatory is operated by Cornell University under contract to the National Science Foundation and with partial support from the Advanced Research Projects Agency. This work was supported in part by the Air Force Office of Scientific Research, Office of Aerospace Research, U.S. Air Force, under contract F44620-69C-0092.

\section{References}

Graham, D. A., Lyne, A. G., and Smith, F. G.: 1970, Nature 225, 526.

Heiles, C., Campbell, D. B., and Rankin, J. M.: 1970, Nature 226, 529.

Heiles, C., and Rankin, J. M.: 1971, submitted to Nature.

Rankin, J. M. and Heiles, C.: 1970, Nature 227, 1330.

Rankin, J. M. and Heiles, C.: 1971b, submitted to Nature.

\section{Discussion}

F. G. Smith: It is important to distinguish between the averages of Stokes parameters over many pulses and the measurements on single pulses. If a single pulse is measured, the radiation is necessarily $100 \%$ elliptically polarised during a time which is the inverse of the receiver bandwidth. A time average 
must be made, which must be over a much longer time. Some of Dr. Heiles' results seem to show an inadequate time smoothing, and without more detail of receiver parameters we should not accept his measurements of pulse structures or circular polarisation.

C. Heiles: The time-bandwidth product for each plotted point $=1.2$. However, there are many cases in which the pulse is, for example, $100 \%$ circularly polarised throughout the whole strong pulse in one bandwidth, while no circular polarisation, or the opposite sense at the $100 \%$ level, occurs in the other band-width which is $200 \mathrm{kHz}$ away. This occurs even for broad strong pulses, for which the effective time-bandwidth product is 4 or 5 . Furthermore, in such cases the Stokes parameter $V$ looks like the total intensity over the whole pulse width, which is clearly improbable if $V$ results simply from statistically random variations in the radiation. 